\title{
Primary sclerosing cholangitis and inflammatory bowel disease comorbidity: an update of the evidence
}

\author{
Andrew Mertza, Nhu An Nguyen ${ }^{b}$, Konstantinos H. Katsanos ${ }^{c}$, Ryan M. Kwok ${ }^{b}$ \\ Walter Reed National Military Medical Center Bethesda, MD, USA; Medical School and University Hospital of \\ Ioannina, Greece
}

\begin{abstract}
Comorbid primary sclerosing cholangitis (PSC) and inflammatory bowel disease (IBD) represent a unique disease phenotype with a different risk profile than PSC or IBD alone. While the pathogenetic mechanisms behind both diseases remain unclear, recent studies have targeted several immune-mediated pathways in an attempt to find a potential therapeutic target. Patients with PSC-associated IBD typically exhibit pancolitis with a right-to-left intestinal inflammatory gradient associated with a greater incidence of backwash ileitis and rectal sparing. Although there is an increased incidence of pancolitis in this population, bowel symptoms tend to be less significant than in IBD alone. Likewise, the degree of inflammation and symptoms of PSC-associated IBD are characteristically less clinically significant. Despite the relatively quiescent clinical presentation of PSC-associated IBD, there is an increased risk for colorectal and hepatobiliary malignancy making vigilance for malignancy essential.
\end{abstract}

Keywords Primary sclerosing cholangitis, inflammatory bowel disease, ulcerative colitis, Crohn's disease

Ann Gastroenterol 2019; 32 (2): 1-10

\section{Introduction}

Inflammatory bowel disease (IBD) and primary sclerosing cholangitis (PSC) are closely associated disease entities that, when present in combination, create a phenotypically different summative disease referred to as PSC-IBD. It is estimated that roughly $70 \%$ of patients with PSC have underlying IBD, most frequently ulcerative colitis (UC) [1]. Conversely, only approximately $5 \%$ of patients with UC will develop PSC [2]. Unfortunately, there is heterogeneity among studies due to a limited understanding of disease pathogenesis. Interestingly, the proportion of patients with PSC complicated with IBD is significantly lower in Japan and Singapore [3] than in North America and Europe, with estimates reported as $20-32 \%[4,5]$ and $60-80 \%$ of PSC cases, respectively [6].

Department of a Internal Medicine (Andrew Mertz); ${ }^{\mathrm{b}}$ Gastroenterology (Nhu An Nguyen, Ryan M. Kwok), Walter Reed National Military Medical Center Bethesda, MD, USA; 'Gastroenterology, Medical School and University Hospital of Ioannina, Greece (Konstantinos H. Katsanos)

Conflict of Interest: None

Correspondence to: Andrew Mertz, 8901 Wisconsin Ave, Bethesda, MD 20889, USA, e-mail: Andrew.t.mertz.mil@mail.mil

Received 17 August 2018; accepted 29 November 2018; published online 15 January 2019

DOI: https://doi.org/10.20524/aog.2019.0344
Although specific pathogenetic mechanisms remain unknown, there are multiple theories that may have clinical significance in the development of novel future therapies. The management of PSC-IBD also differs from that of PSC or IBD alone, owing to differences in disease manifestation and malignancy risk. Despite practice guidelines for the management of PSC and IBD, there is a paucity of supporting data in PSC-IBD to make firm, evidence-based recommendations for managing these patients. This is of particular importance, as an increased risk of malignancy is one of the hallmarks of PSC-IBD [6-9]. The purpose of this review is to discuss the unique disease phenotype, explore the potential mechanisms behind PSC-IBD, and review the increased risk and screening for malignancy in this unique patient population.

\section{Characteristic features of IBD associated with PSC}

The initial association between PSC and IBD was first described in 1965 by Smith and Loe [10]. Since that time, studies of population-based cohorts and meta-analyses have investigated the exact prevalence and sequelae of PSC-IBD. Recently, PSC-IBD has been described as a unique entity characterized by an increased risk for colorectal cancer (CRC) and relatively quiescent colitis $[11,12]$. Globally, the prevalence of IBD in modernized countries is estimated at $0.3 \%$, based on population data from a 2017 meta-analysis [13]. While the 
exact worldwide prevalence of PSC-IBD remains unknown, because of the varying diagnostic criteria, it is best estimated at $0.024 \%$ [14]. Within the central and southern European population, there are limited data regarding the prevalence of PSC-IBD. Extrapolation of population data from Hungary estimates the prevalence of PSC-IBD in this region to be $0.041 \%[15,16]$. Prior research confounds these data, as the incidence of extraintestinal manifestations of IBD is lower in southern Europe, particularly northwest Greece [17].

\section{PSC-UC}

UC accounts for approximately $80 \%$ of IBD cases in PSCIBD patients, with Crohn's disease (CD) and indeterminate colitis each representing $10 \%$ [12]. For this reason, most studies compare the clinical course, risk for malignancy and management of PSC-IBD to UC. The clinical presentation and course of PSC-IBD vary. Characteristically, colitis tends to be more quiescent and may even appear endoscopically normal in patients with active colitis on biopsy [1]. This makes diagnosis particularly challenging and informs recommendations for random biopsies during initial diagnostic colonoscopy. Likewise, cholangitis can be asymptomatic and may go undiagnosed in patients with known IBD. For this reason, current recommendations include screening for PSC in IBD patients with annual liver function tests, regardless of symptoms. Fig. 1 depicts a proposed algorithm for PSC screening and diagnosis in patients with IBD. In these patients, the annual liver function should be assessed for cholestatic and hepatocellular patterns of injury. It is important to note that this same liver function screening interval is still recommended in UC patients who have undergone colectomy, as they are still at increased risk for PSC compared with the general population. If a cholestatic pattern arises (see Fig. 1 for R-factor details), then imaging with cholangiography is indicated [18]. If a patient with IBD is found to have a hepatocellular injury pattern on routine liver function testing, then an appropriate workup should be completed to explore the potential etiology. If this workup is unrevealing, then it is reasonable to explore other less common etiologies, such as PSC. Regarding the diagnostic modalities for PSC, magnetic resonance cholangiopancreatography (MRCP) is preferred over endoscopic retrograde cholangiopancreatography (ERCP), because it has been shown to have good diagnostic accuracy and is not associated with the risks that ERCP entails [19]. As in the routine hepatic screening done for IBD patients, PSC patients should be screened for IBD at the time of initial diagnosis then followed by close-interval colonoscopy [1]. The algorithm found in Fig. 1 is based, in part, on expert opinion, and should not be used in place of clinical judgement.

The data regarding the chronology of presentation for PSC-IBD are also heterogeneous. Most data indicate that IBD typically precedes PSC, with a median time interval of 10 years [20]. In contrast, other studies have noted an increased incidence of PSC after colectomy in IBD patients [21]. Furthermore, there are also reports of IBD appearing many

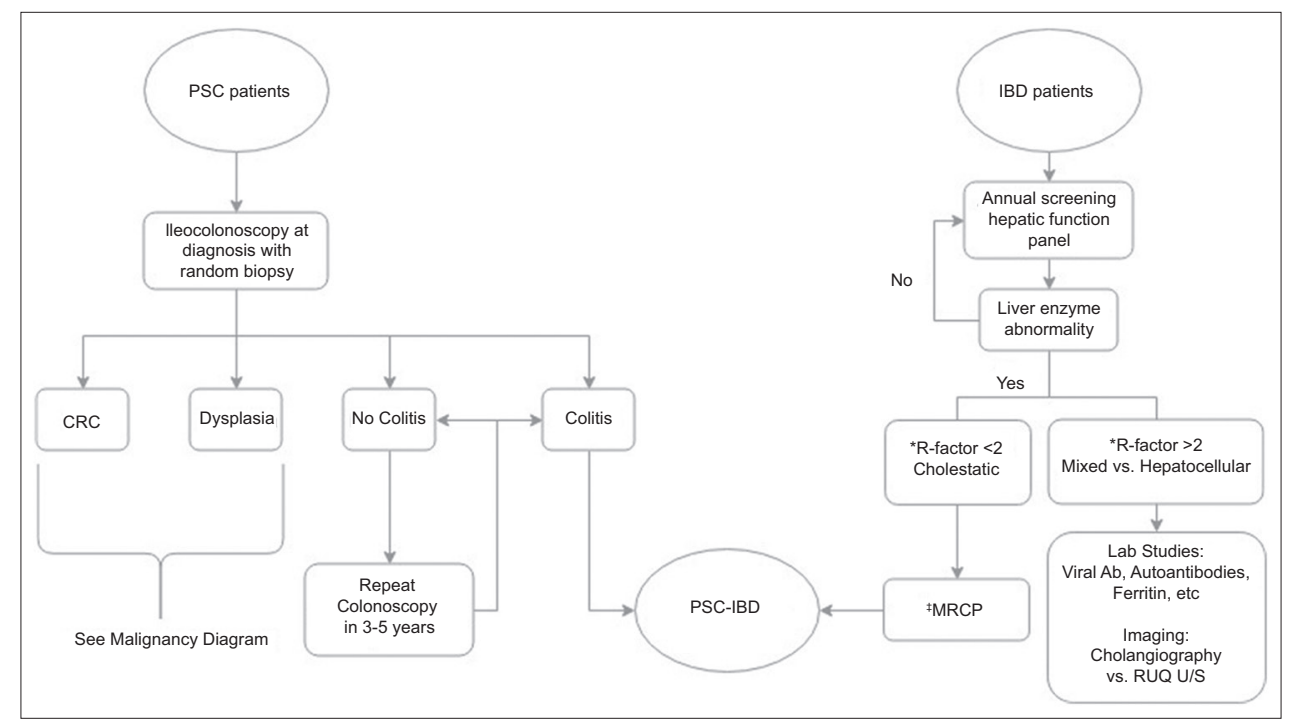

Figure 1 PSC-IBD diagnostic algorithm

${ }^{\star} \mathrm{R}$-factor is defined as the ratio of alanine aminotransferase (ALT) to alkaline phosphatase (ALP) elevation as compared to the upper limit of normal (ULN). The following equation can be used to obtain this value: R-factor= (ALT/ALTULN) $\div($ ALP/ ALPULN). If the quotient is less than 2, then it suggests a cholestatic process. A quotient of 2-5 suggests a mixed process and values greater than 5 suggest a hepatocellular injury pattern [18]

¥MRCP is the preferred ima ging modality for diagnosis of PSC as it has good diagnostic accuracy and does not carry the potential risks associated with endoscopic retrograde cholangiopancreatography [19]

PSC, primary sclerosing cholangitis; IBD, inflammatory bowel disease; MRCP, magnetic resonance cholangiopancreatography; $C R C$, colorectal cancer; $A b$, antibody; RUQ, right upper quadrant; U/S, ultrasound [58,78-80] 
years after the diagnosis of PSC, or even after orthotopic liver transplantation [22]. Given this uncertainty, clinicians should remain vigilant for PSC-IBD in patients with known PSC or IBD.

The distribution of disease and the degree of endoscopic inflammation seen in PSC-IBD set it apart from IBD alone. Multiple studies have demonstrated an increased incidence of pancolitis among patients with PSC-UC compared to UC-only patients [12,23-26]. In a study of almost 400 patients with PSCIBD, Boonstra et al reported that $94 \%$ of the PSC-UC patients had pancolitis, compared with only $62 \%$ in the matched UC-only cohort $(\mathrm{P}<0.001)$ [24]. Although the distribution of colitis appears to be more diffuse, the clinical course in PSC-IBD appears to be less severe. Several studies note that PSC-IBD patients require fewer steroid equivalents and fewer hospital admissions than their IBD counterparts [26,27]. Backwash ileitis and rectal sparing are two characteristics initially thought to be more prevalent in PSC-IBD than in IBD alone. However, significant heterogeneity found in recently pooled data calls this claim into question. The mild degree of macroscopic, endoscopic inflammation may underestimate the degree of rectal inflammation, leading to the appearance of increased rectal sparing in PSC-IBD. Further investigation into the macroscopic disease characteristics, such as backwash ileitis and rectal sparing, are clinically relevant, as they may aid in pattern recognition and diagnosis [12,24,28].

\section{PSC-CD}

Similarly to PSC-UC, the PSC-CD phenotype differs significantly from that of classic CD. Specifically, isolated ileal involvement in PSC-CD is rare, with rates ranging from $2-5 \%$, compared with rates of around $30 \%$ in typical CD [24,29-31]. Thus, the rates of colitis and ileocolitis are higher in PSC-CD than in CD alone [15]. As with PSC-UC, the degree of macroscopic inflammation seen on endoscopy is milder than that in controls, which may account for the lower rates of fibrostenotic and penetrating disease seen in PSCCD patients $[24,29,30]$. The apparently lower rates of isolated ileal involvement, fibrostenotic and penetrating disease seen in PSC-CD do, however, raise the question whether many of these patients are misdiagnosed because of the wide variability in CD clinical presentations.

\section{Characteristics of PSC associated with IBD}

While the presence of IBD with PSC appears to convey a specific IBD phenotype, the converse does not appear to be true. Microscopically, an early study noted no significant difference in the degree of histologic periductal fibrosis, periductal inflammation, portal edema or fibrosis in PSC vs. PSC-UC patients [32]. Macroscopically, data conflict regarding the degree of intrahepatic and extrahepatic involvement in PSC-IBD vs. PSC alone [33-35]. Clinically, PSC outcome measurements, including transplant-free survival rates, cirrhosis rates and mortality, did not differ significantly between PSC-IBD patients and those with PSC-alone [35]. Notably, however, recent studies by Fevery and Weismüller report higher rates of liver-related morbidity and mortality in PSC-UC patients when compared to patients with PSC-CD or PSC alone. These data suggest the subtype of IBD may have an impact on the course of PSC disease progression [36,37].

Given the unique phenotype of PSC-IBD, it has been postulated that the disease activity present in each organ may have an impact on the other. Recent research into the relationship between IBD disease activity and PSC outcomes has revealed that colectomy in particular was an intervention that appeared to have an impact on hepatic disease activity. Two studies published in 2018, probably with overlapping patient population data, specifically looked at the impact of colectomy on PSC-IBD patients. Nordenvall et al found that early colectomy, even prior to the diagnosis of PSC in over 200 patients, was associated with a decreased risk for future liver transplantation and mortality [21]. Furthermore, Lindström et al found that PSC-IBD patients treated with colectomy prior to liver transplantation were at decreased risk for recurrent PSC. However, it is worth noting that in this study the IBD disease activity was not associated with the risk of recurrent PSC in the hepatic graft [38]. These two studies present compelling data that hint towards a potential disease mechanism that could potentially be exploited with future research.

\section{Potential mechanisms for disease pathogenesis}

The recognition of the unique PSC-IBD phenotype has stimulated research into potential pathogenic mechanisms. A variety of mechanisms have been proposed in an effort to determine a unifying pathway for this unique phenotype.

\section{Autoantibodies}

Autoantibodies are a classic biomarker associated with both PSC and IBD. Perinuclear anti-neutrophil cytoplasmic antibodies (pANCA) are one such marker classically related to both of these diseases. However, this association may not be as strong as previously thought, as the prevalence in the sera of patients with PSC in the absence of IBD has been estimated to be $25-77 \%$ compared with the rates of $23-88 \%$ in PSCIBD patients [39]. While the exact pathogenetic role for these antibodies remains unknown, there have been several recent studies examining the role of PANCA in both PSC and IBD. In PSC, the molecular targets of pANCA are both lactoferrin and tubulin-beta isotope $5[40,41]$, whereas in UC nuclear histone is the target, suggesting a differing role for this antibody between diseases [42]. Interestingly, the immunoglobulin (IgG) subclasses of pANCA have also been studied to determine if a higher expression of a particular isotype within PSC and UC exists. Targan et al found the predominant subtype of pANCA 
in PSC to be IgG1 and IgG3, while Seibold et al noted IgG1 as predominant in UC $[43,44]$ suggesting that pANCA IgG1 could be a pathogenic link. However, IgG1 is not unique to PSC and $\mathrm{UC}$, as it is also expressed in autoimmune hepatitis [43].

While pANCA may not be the sole link between PSC and IBD, it may serve as a measure for predicting disease activity in these diseases. Roozendaal et al found that in patients with PSC the presence of ANCA was associated with a decreased serum albumin and increased alkaline phosphatase, suggestive of a more severe disease activity [40]. Additional studies demonstrate the relapse rate in pANCA-positive UC patients vs. controls potentially indicating a prognostic role for this assay [45]. Likewise, the presence of pANCA in CD appears to confer a clinical phenotype resembling UC and is prognostically indicative of severe disease in the 5-year period following diagnosis [46,47]. Given that the presence of pANCA in PSC patients appears to be indicative of severe disease and the presence in IBD patients portends a worse prognosis, a common autoantibody may link the two diseases.

\section{Genetics}

Despite initial enthusiasm for a genetic link in PSC-IBD, recent genomics data have yet to find a strong association. Genome-wide association studies have identified over 150 regions of interest in IBD loci common to UC and CD [48], while other studies identified 9 susceptibility loci associated with the development of PSC [49]. Unfortunately, there was limited overlap between the two groups of susceptibility loci [50]. Nonetheless, it is hypothesized that a specific population of patients may be genetically predisposed to autoimmune biliary injury triggered by colonic inflammation.

\section{Bile acid (BA) composition}

BA and microbiome interaction component of IBD has also been recently studied and there are data suggesting that some of the BA products seen in IBD patients as a result of gut dysbiosis have a potential proinflammatory effect [50]. Secondary BAs are considered anti-inflammatory in nature, as they have been shown in the literature to inhibit the release of tumor necrosis factor- $\alpha$, interleukin (IL)-1B and IL-6 from in vitro macrophages $[51,52]$. More recently, secondary BAs, such as lithocholic acid, have been found to have an anti-inflammatory dose-response relationship by inhibiting IL-8 secretion [50]. The researchers also found that the concentration of secondary BAs within the stool was inversely proportional to the disease activity, with high concentrations in the stool from control subjects and low concentrations in the stool from patients with active IBD [50]. While it is unknown whether the inflammatory state causes dysbiosis, resulting in decreased secondary BA production, or the secondary BAs themselves are anti-inflammatory in vivo, there does appear to be a strong association between secondary BA concentration and inflammation. Although prior studies have praised secondary BAs for their anti-inflammatory properties, more recent studies have found that carcinogenesis is also part of the effect profile for these molecules. One study used an animal model to determine that a diet enriched in deoxycholic acid, a secondary BA, induced colonic tumor formation in 17 of the 18 mice in the study [53]. Moreover, a review paper discussed that BAs may cause DNA damage in vitro and select for apoptosisresistant cells, indicating their carcinogenic potential [54].

Recently, there has been specific interest regarding the importance of BAs in the progression of disease in IBD and PSC-IBD. Much like the Duboc et al study completed years earlier, a 2018 study of 30 IBD patients (15 with concomitant PSC) found that there was a negative correlation between the stool concentration of secondary BAs and endoscopic disease activity. Regarding PSC-IBD specifically, the study found that there was no significant difference between the concentration of secondary BAs between the PSC-IBD and IBD subgroups [55]. While BA concentrations and host microbiome-BA interaction appear to have a significant role in intestinal inflammation, the most recent data do not appear to have revealed a mechanism that can be applied specifically to PSC-IBD patients.

\section{The "lymphocyte-homing" model}

Theories aimed toward establishing immunologic ties between the two diseases have been proposed more recently. The first of these hypotheses proposes that intestinal mucosal lymphocytes home to the liver following activation in the bowel of IBD patients [56]. Clinically, this theory is supported by the observation that PSC may develop after total colectomy in IBD patients and that the degree of hepatic disease does not correlate well with the intestinal disease activity of IBD $[57,58]$. On a molecular level, this theory is supported by powerful data showing that a network of adhesion molecules and chemokine receptors normally restricted to the gut can be aberrantly expressed within the liver to promote the homing to gut-associated lymphocytes through the enterohepatic circulation. The most widely recognized adhesion molecule in this family is $\alpha 4 \beta 7$ integrin, also known as lymphocyte Peyer's patch adhesion molecule 1 . The a $4 \beta 7$ monoclonal antibody, vedolizumab, has been approved for the treatment of IBD. It was hypothesized that, as $\alpha 4 \beta 7+$ lymphocytes infiltrate the liver in PSC, the monoclonal antibody for this integrin molecule could potentially provide hepatic anti-inflammatory benefits. Christensen et al found that, although the clinical activity of intestinal disease was significantly decreased after treatment with vedolizumab, unfortunately the liver biochemistry and the Mayo PSC Risk Score were only slightly impacted on PSCIBD [59].

Another of these adhesion molecules, mucosal addressing cell adhesion molecule (MAdCAM-1), was studied specifically by Grant et al back in 2001. They found that MAdCAM-1 expression on hepatic vessels supported the adhesion of gutderived $\alpha 4 \beta 7+$ lymphocytes, demonstrating that rolling adhesion was inhibited when tissue was pre-treated with MAdCAM-1 or $\alpha 4 \beta 7$ monoclonal antibodies [60]. The phase 2, double- 
blind randomized, placebo-controlled trial for MAdCAM-1 monoclonal antibody (PF-00547659) was recently published in 2017 and found that the drug was better than placebo for the induction of remission in patients with moderate-to-severe UC [61]. The effects of this monoclonal antibody in patients with PSC-IBD are yet to be studied in vivo, but extrapolation of data from the in vitro study by Grant et al implies that perhaps treatment with both of these antibodies could impair the adhesion of the gut-associated lymphocytes responsible for hepatic inflammation in PSC. The aberrant expression of MAdCAM-1 and chemokine ligand 25 within the liver in PSC remains poorly understood, but with the new advent of monoclonal antibodies targeted at inhibiting the interaction of these molecules with gut-derived lymphocytes, there could potentially be good utility in the treatment of PSC-IBD.

\section{The "leaky gut" model}

Another hypothesized mechanism behind PSC-IBD is the "leaky gut" hypothesis, which states that the chronic inflammation within the gut causes increased permeability of the intestinal mucosa. This renders it susceptible to bacterial and bacterial product translocation that infiltrates the enterohepatic circulation and results in hepatic inflammation [62]. Several factors support this theory, such as the fact that $75 \%$ of the blood supply to the liver comes from the splanchnic circulation, which effectively exposes the liver to all the noxious products from the gut that may invade the enterohepatic circulation [63]. Through this enterohepatic circulation, gram-negative and anaerobic bacterial components such as peptidoglycan polysaccharide or chemotactic peptides gain access to the liver and have been shown to create hepatic lesions reminiscent of PSC in rat models [64]. However, there appear to be preliminary data arguing for the contrary. Specifically, a randomized placebo-controlled trial published in 2004 found that, although metronidazole and ursodeoxycholic acid had a favorable effect on serum alkaline phosphatase levels and Mayo Risk Score in patients with PSC, the medicines did not significantly affect disease progression and thus probably do not decrease the quantity of noxious epitopes entering the enterohepatic circulation. However, the authors did note that the trial was significantly limited by the small sample size and lack of long-term follow up [65].

\section{Gut dysbiosis}

Rather than implicating certain pathogenic bacteria in IBD and PSC, gut dysbiosis and decreased microbial diversity has been the new target for research [50]. Recent studies aimed towards discovering discrepancies between the gut microbiome of IBD and PSC-IBD patients have noted an increase in Escherichia, Fusobacterium, Ruminococcus and Megaspera genera in PSC-IBD patients. These same studies also found that other genera, including Prevotella, Roseburia, Dorea, Blautia and Bacterioides are reduced [55,66-68]. Interestingly, Veillonella and Lachnospira genera trends in PSC-IBD patients do not appear to be uniform across the studies. While there are limited data regarding the importance of Lachnospira in IBD, the literature does report the impact of increased Veillonella on IBD. A 2017 study found that increased Veillonella was associated with more significant PSC disease severity and was found more frequently in patients who had already undergone orthotopic liver transplantation [67]. Interestingly, the Veillonella genus has also been implicated in other fibrotic conditions, such as cirrhosis and lung fibrosis [69-71]. Given the conflicting data regarding the presence of Veillonella in the microbiome of PSCIBD patients, it is unclear whether the presence of this genus is a cause or a result of the disease process; nevertheless, it brings to light how complicated the relationship between these two diseases may be. Further studies with a larger sample size may potentially help elucidate whether this genus is at all related to the pathogenesis of PSC-IBD.

\section{Risk of malignancy in PSC-IBD}

There is a high risk of malignancy in both PSC and IBD alone. However, their combination confers a significantly greater risk and requires shorter screening intervals for both CRC and hepatobiliary malignancy. Unfortunately, because of the relatively recent description of this phenotype, there is no

Table 1 Risk of CRC in PSC-IBD compared with IBD patients based on cohort and meta-analysis data

\begin{tabular}{lllll}
\hline UC & CD & PSC-UC & PSC-UC & PSC-IBD \\
\hline Eaden et al (2001) [76] & Canavan et al (2006) [77] & Kornfeld et al (1997) [7] & Soetikno et al (2002) [8] & Zheng et al (2016) [9] \\
Meta-analysis 116 studies & Meta-analysis 14 studies & Population cohort & Meta-analysis 11 studies & Meta-analysis 16 studies \\
54,478 UC patients & 11,545 CD patients & 125 PSC-UC patients & 564 PSC-UC patients & 1,022 PSC-IBD patients \\
USA, UK, Scandinavia, & USA, Denmark, UK, & Sweden & USA, Finland, Sweden, & USA, Scandinavia, \\
Other & Sweden, Canada, Israel & & Germany & Argentina, UK, France, \\
& & & Netherlands \\
Cumulative risk of CRC & Cumulative risk of CRC & Cumulative risk & OR for CRC & OR for CRC \\
$1.6 \%$ at 10 years & $2.9 \%$ at 10 years & $10 \%$ at 10 years & $4.79(3.58-6.41)$ & $3.24(2.14-4.90)$ \\
$8.3 \%$ at 20 years & $5.6 \%$ at 20 years & $33 \%$ at 20 years & & \\
$18.4 \%$ at 30 years & $8.3 \%$ at 30 years & $40 \%$ at 30 years & & \\
\hline
\end{tabular}

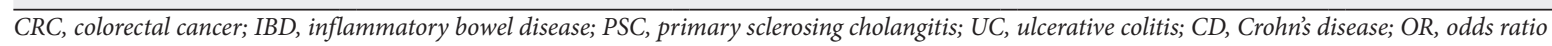


strong evidence to inform malignancy screening guidelines. As a result, several of the screening recommendations are from guidelines describing PSC and IBD alone and are extrapolated to include screening in PSC-IBD patients.

\section{CRC screening}

In IBD, the risk of CRC is greatest with pancolitis and is further increased with higher degrees of endoscopic and histological inflammation [72,73]. Numerous studies demonstrated a significantly increased risk for CRC or dysplasia in patients with PSC-IBD [7-9,74-77] compared with IBD alone (Table 1).

Given the strong association between CRC and PSC-IBD, providers should be vigilant with CRC screening in PSC-IBD patients, regardless of their IBD subtype. Consensus guidelines for IBD diagnosis and CRC screening are summarized in Table 2. Despite some small differences in their screening recommendations, it is worth noting that each major American and European Society recommends that annual CRC screening be started at the time of PSC-IBD diagnosis [58,78-80]. There are a few minor differences between the guidelines regarding screening intervals and endoscopic modalities. With regard to the diagnosis of IBD in patients with known PSC, all of the societies recommend random biopsies be performed during endoscopy. Chromoendoscopy is also recommended by other societies' guidelines, apart from those of the American Association for the Study of Liver Diseases (AASLD), which do not comment on the use of chromoendoscopy. In PSC patients without colitis on their initial screening endoscopy, the European Crohn's and Colitis Organisation, the European Association for the Study of the Liver and the American College of Gastroenterology (ACG) recommend a 3-5-year interval for repeat endoscopy, or sooner if symptoms consistent with colitis emerge [58,78-80].

\section{Hepatobiliary carcinoma screening}

Unfortunately, the increased risk for malignancy in PSCIBD is not isolated to CRC and is also seen in other hepatobiliary malignancies such as cholangiocarcinoma (CCA), gallbladder carcinoma (GBC), and hepatocellular carcinoma (HCC). In a study of nearly 400 PSC-IBD patients, Gulamhusein et al noted an increased risk for CCA in patients with a prolonged duration of IBD [81]. As stated above, there are no society consensus guidelines on malignancy screening explicitly for PSC-IBD patients. Table 3 shows the recommended screening tests and intervals for patients with PSC alone from each of the major American and European professional societies. With regard to CCA they all appear to agree that screening CA 19-9 and biliary imaging should be completed annually $[59,78,80,82]$.

While no formal studies have been completed aimed towards determining an increased risk for GBC in the PSCIBD population, there are recommendations regarding the management of gallbladder polyps in PSC-alone patients. As there are no widely accepted guidelines for malignancy screening in PSC-IBD patients, clinical judgement must be used to create an individualized care plan for each patient. Although there is no consensus about when to perform cholecystectomy in PSC patients with gallbladder polyps,

Table 2 Societal guidelines for diagnostic and CRC screening endoscopy in PSC patients

\begin{tabular}{lllll}
\hline Variable & ECCO 2017 [78] & EASL 2017 [79] & ACG 2015 [80] & AASLD 2010 [58] \\
\hline Ileocolonoscopy at PSC diagnosis & Recommended & Recommended & Recommended & Recommended \\
Random biopsy & Recommended & Recommended & Recommended & Recommended \\
Preferred modality & Chromoendoscopy & Chromoendoscopy & Chromoendoscopy & Colonoscopy \\
Screening interval & & & & \\
With colitis & Annually & Annually & Annually & Every 1-2 years \\
Without colitis & Every 3-5 years & Every 5 years & Every 3-5 years & No recommendation \\
\hline
\end{tabular}

CRC, colorectal cancer; PSC, primary sclerosing cholangitis; ECCO, European Crohn's and Colitis Organisation; EASL, European Association for the Study of the Liver; ACG, American College of Gastroenterology; AASLD, American Association for the Study of Liver Diseases

Table 3 Recommendations regarding cholangiocarcinoma and gallbladder carcinoma screening in patients with PSC alone

\begin{tabular}{lllll}
\hline & ECCO 2017 [78] & EASL 2009 [79] & ACG 2015 [80] & AASLD 2010 [58] \\
\hline CCA screening tests & Ultrasound, & Ultrasound or MRI, & Ultrasound or MRI, & Ultrasound or MRI, \\
& CA 19-9 & CA 19-9 & CA 19-9 & CA 19-9 \\
CCA screening interval & Annually & Annually & Every 6-12 months & Annually \\
Cholecystectomy & $\begin{array}{l}\text { No } \\
\text { recommendations }\end{array}$ & $\begin{array}{l}\text { For any GB mass, } \\
\text { even }<1 \mathrm{~cm}\end{array}$ & GB polyps $>8$ mm & $\begin{array}{l}\text { For any GB mass, } \\
\text { even }<1 \mathrm{~cm}\end{array}$ \\
\hline
\end{tabular}

CCA, cholangiocarcinoma; PSC, primary sclerosing cholangitis; ECCO, European Crohn's and Colitis Organisation; EASL, European Association for the Study of the Liver; ACG, American College of Gastroenterology; AASLD, American Association for the Study of Liver Diseases; GB, gallbladder; MRI, magnetic resonance imaging 


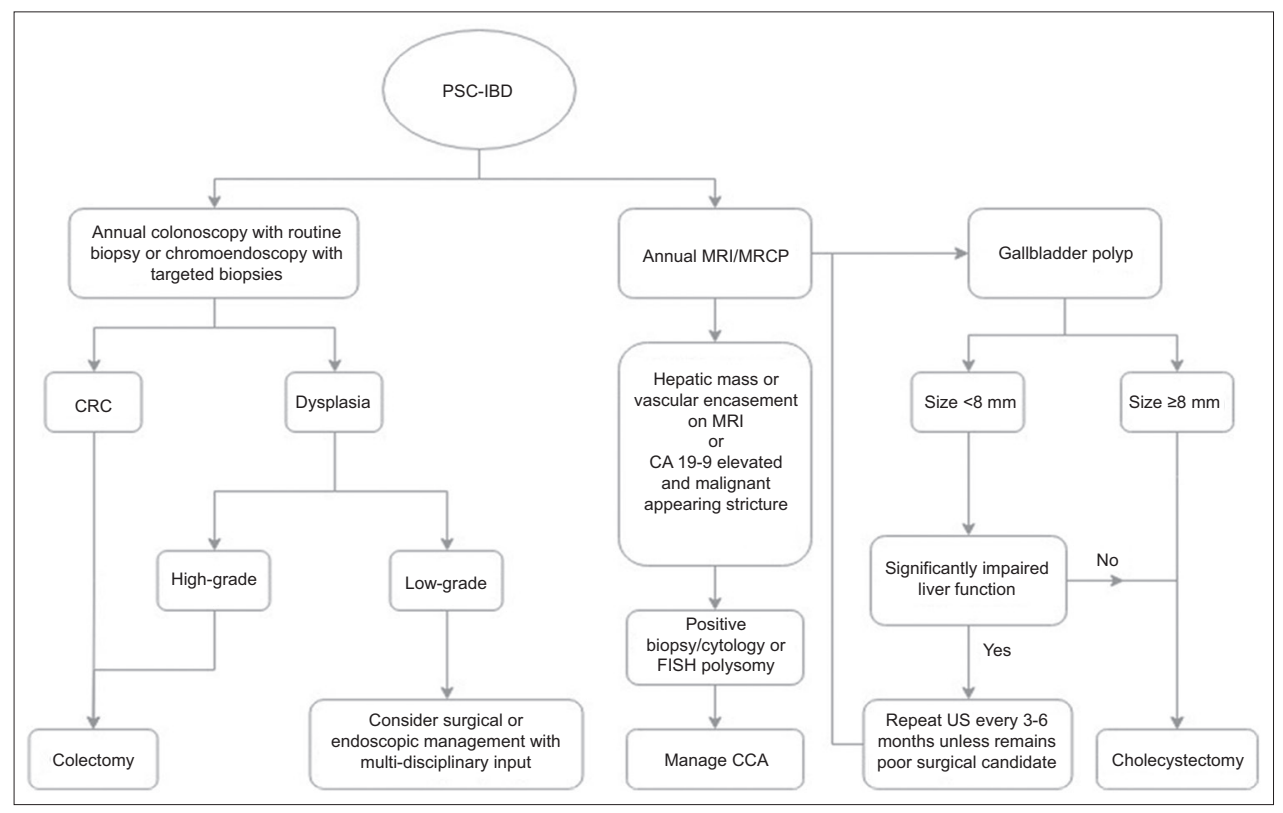

Figure 2 PSC-IBD malignancy screening algorithm

PSC, primary sclerosing cholangitis; IBD, inflammatory bowel disease; MRI, magnetic resonance imaging; MRCP, magnetic resonance cholangiopancreatography; CRC, colorectal cancer; CCA, cholangiocarcinoma; FISH, fluorescence in situ hybridization; US, ultrasound [58,78-80,85, 86]

AASLD guidelines support cholecystectomy for polyps of any size in these patients, given the high likelihood of malignancy. More recent data suggest that using a polyp size cutoff of $>8 \mathrm{~mm}$ achieves $100 \%$ sensitivity and $70 \%$ specificity for GBC in PSC patients $[58,83]$. This is of particular importance, as the leading cause of death in PSC patients undergoing cholecystectomy is liver-related complications [83].

In contrast to colorectal and gallbladder and biliary malignancies, HCC appears to be relatively rare in PSC-IBD. While there have been no studies formally evaluating the incidence of HCC in PSC-IBD, there are recent data regarding HCC rates in PSC alone. Zenouzi et al analyzed 292 patientyears of data from over 100 patients with PSC cirrhosis and found no cases of HCC, even on liver explant. These findings suggest that HCC is likely a rare sequela of PSC and may offer clues to the PSC-IBD population, as roughly $65 \%$ of the patients enrolled in the study had comorbid IBD [84].

Fig. 2 represents our suggested algorithm for malignancy screening in PSC-IBD patients. Owing to the lack of formal guidelines, malignancy screening must be based on individual risk factors and tailored to specific patients. Treatment options for high-grade and low-grade dysplasia have been integrated into the screening colonoscopy arm, based on interventions proposed by prior literatures $[85,86]$. While there is no consensus guideline regarding HCC screening in patients with PSC-IBD specifically, the ACG's PSC clinical practice guidelines recommend that imaging via ultrasound or magnetic resonance and CA 19-9 be performed every 6-12 months. It is important to note that these recommendations are for all PSC patients, even those without cirrhosis, and are made with a very low quality of evidence [80].

\section{Concluding remarks}

With improved understanding of the mechanisms and characteristics of both PSC and IBD, PSC-IBD is likely to gain new treatment and guideline updates in the coming years. The characteristics of the PSC-IBD phenotype are clinically relevant, as they aid in both the diagnosis and management of this patient population. PSC-IBD is typically associated with pancolitis with a right-sided predilection, as well as potential rectal sparing and backwash ileitis. Furthermore, endoscopic inflammation is generally milder and associated with less severe symptoms. There is a significantly increased risk of malignancy associated with PSC-IBD, including CRC, $\mathrm{CCA}$, and GBC. Therefore, clinicians must remain vigilant for malignancy in these patients. Although there have been exciting developments in the understanding of this disease, the pathogenetic mechanisms remain unknown. Currently, the diseases are thought to be influenced by lymphocytic crossreactivity, aberrant microbiotic epitope recognition and gut dysbiosis. New biologic agents targeted towards altering the interaction between the immune system and the target organs may provide novel therapies for PSC-IBD.

\section{References}

1. de Vries AB, Janse M, Blokzijl H, Weersma RK. Distinctive inflammatory bowel disease phenotype in primary sclerosing cholangitis. World J Gastroenterol 2015;21:1956-1971.

2. Rasmussen HH, Fallingborg JF, Mortensen PB, Vyberg M, Tage- 
Jensen U, Rasmussen SN. Hepatobiliary dysfunction and primary sclerosing cholangitis in patients with Crohn's disease. Scand J Gastroenterol 1997;32:604-610.

3. Nakazawa T, Naitoh I, Hayashi K, et al. Inflammatory bowel disease of primary sclerosing cholangitis: a distinct entity? World J Gastroenterol 2014;20:3245-3254.

4. Takikawa H, Takamori Y, Tanaka A, Kurihara H, Nakanuma Y. Analysis of 388 cases of primary sclerosing cholangitis in Japan; presence of a subgroup without pancreatic involvement in older patients. Hepatol Res 2004;29:153-159.

5. Ang TL, Fock KM, Ng TM, Teo EK, Chua TS, Tan JY. Clinical profile of primary sclerosing cholangitis in Singapore. J Gastroenterol Hepatol 2002;17:908-913.

6. Eaton JE, Talwalkar JA, Lazaridis KN, Gores GJ, Lindor KD. Pathogenesis of primary sclerosing cholangitis and advances in diagnosis and management. Gastroenterology 2013;145:521-536.

7. Kornfeld D, Ekbom A, Ihre T. Is there an excess risk for colorectal cancer in patients with ulcerative colitis and concomitant primary sclerosing cholangitis? A population based study. Gut 1997;41:522-525.

8. Soetikno RM, Lin OS, Heidenreich PA, Young HS, Blackstone MO. Increased risk of colorectal neoplasia in patients with primary sclerosing cholangitis and ulcerative colitis: a meta-analysis. Gastrointest Endosc 2002;56:48-54.

9. Zheng HH, Jiang XL. Increased risk of colorectal neoplasia in patients with primary sclerosing cholangitis and inflammatory bowel disease: a meta-analysis of 16 observational studies. Eur J Gastroenterol Hepatol 2016;28:383-390.

10. Smith MP, Loe RH. Sclerosing cholangitis; review of recent case reports and associated diseases and four new cases. Am J Surg 1965;110:239-246.

11. Loftus EV Jr, Sandborn WJ, Tremaine WJ, et al. Risk of colorectal neoplasia in patients with primary sclerosing cholangitis. Gastroenterology 1996;110:432-440.

12. Loftus EV Jr, Harewood GC, Loftus CG, et al. PSC-IBD: a unique form of inflammatory bowel disease associated with primary sclerosing cholangitis. Gut 2005;54:91-96.

13. Ngu JH, Gearry RB, Wright AJ, Stedman CA. Inflammatory bowel disease is associated with poor outcomes of patients with primary sclerosing cholangitis. Clin Gastroenterol Hepatol 2011;9:1092-1097.

14. Lunder AK, Hov JR, Borthne A, et al. Prevalence of sclerosing cholangitis detected by magnetic resonance cholangiography in patients with long-term inflammatory bowel disease. Gastroenterology 2016;151:660-669.

15. Lakatos L, Kiss LS, David G, et al. Incidence, disease phenotype at diagnosis, and early disease course in inflammatory bowel diseases in Western Hungary, 2002-2006. Inflamm Bowel Dis 2011;17:2558-2565.

16. Burisch J, Jess T, Martinato M, Lakatos PL; ECCO -EpiCom. The burden of inflammatory bowel disease in Europe. J Crohns Colitis 2013;7:322-337.

17. Katsanos KH, Stamou P, Tatsioni A, et al; Northwest Greece IBD Study Group. Prevalence of inflammatory bowel disease related dysplasia and cancer in 1500 colonoscopies from a referral center in northwestern Greece. J Crohns Colitis 2011;5:19-23.

18. Chalasani NP, Hayashi PH, Bonkovsky HL, Navarro VJ, Lee WM, Fontana RJ; Practice Parameters Committee of the American College of Gastroenterology. ACG Clinical Guideline: the diagnosis and management of idiosyncratic drug-induced liver injury. Am J Gastroenterol 2014;109:950-966.

19. Dave M, Elmunzer BJ, Dwamena BA, Higgins PD. Primary sclerosing cholangitis: meta-analysis of diagnostic performance of MR cholangiopancreatography. Radiology 2010;256:387-396.

20. Sørensen J, Nielsen OH, Andersson M, et al. Inflammatory bowel disease with primary sclerosing cholangitis: a Danish populationbased cohort study 1977-2011. Liver Int 2018;38:532-541.

21. Nordenvall C, Olén O, Nilsson PJ, et al. Colectomy prior to diagnosis of primary sclerosing cholangitis is associated with improved prognosis in a nationwide cohort study of 2594 PSC-IBD patients. Aliment Pharmacol Ther 2018;47:238-245.

22. Riley TR, Schoen RE, Lee RG, Rakela J. A case series of transplant recipients who despite immunosuppression developed inflammatory bowel disease. Am J Gastroenterol 1997;92:279-282.

23. Joo M, Abreu-e-Lima P, Farraye F, et al. Pathologic features of ulcerative colitis in patients with primary sclerosing cholangitis: a case-control study. Am J Surg Pathol 2009;33:854-862.

24. Boonstra K, van Erpecum KJ, van Nieuwkerk KM, et al. Primary sclerosing cholangitis is associated with a distinct phenotype of inflammatory bowel disease. Inflamm Bowel Dis 2012;18:2270-2276.

25. Shiau H, Ihekweazu FD, Amin M, Fofanova T, Miloh T, Kellermayer R. Unique inflammatory bowel disease phenotype of pediatric primary sclerosing cholangitis: a single-center study. J Pediatr Gastroenterol Nutr 2017;65:404-409.

26. Shah SC, Ten Hove JR, Castaneda D, et al. High risk of advanced colorectal neoplasia in patients with primary sclerosing cholangitis associated with inflammatory bowel disease. Clin Gastroenterol Hepatol 2018;16:1106-1113.

27. Sano H, Nakazawa T, Ando T, et al. Clinical characteristics of inflammatory bowel disease associated with primary sclerosing cholangitis. J Hepatobiliary Pancreat Sci 2011;18:154-161.

28. Ricciuto A, Fish J, Carman N, et al. Symptoms do not correlate with findings from colonoscopy in children with inflammatory bowel disease and primary sclerosing cholangitis. Clin Gastroenterol Hepatol 2018;16:1098-1105.

29. Lindström L, Lapidus A, Ost A, Bergquist A. Increased risk of colorectal cancer and dysplasia in patients with Crohn-s colitis and primary sclerosing cholangitis. Dis Colon Rectum 2011;54:1392-1397.

30. Halliday JS, Djordjevic J, Lust M, et al. A unique clinical phenotype of primary sclerosing cholangitis associated with Crohn's disease. J Crohns Colitis 2012;6:174-181.

31. Freeman HJ. Application of the Montreal classification for Crohn's disease to a single clinician database of 1015 patients. Can J Gastroenterol 2007;21:363-366.

32. Ludwig J, Barham SS, LaRusso NF, Elveback LR, Wiesner RH, McCall JT. Morphologic features of chronic hepatitis associated with primary sclerosing cholangitis and chronic ulcerative colitis. Hepatology 1981;1:632-640.

33. Rabinovitz M, Gavaler JS, Schade RR, Dindzans VJ, Chien MC, Van Thiel DH. Does primary sclerosing cholangitis occurring in association with inflammatory bowel disease differ from that occurring in the absence of inflammatory bowel disease? A study of sixty-six subjects. Hepatology 1990;11:7-11.

34. Navaneethan U, Venkatesh PG, Lashner BA, Shen B, Kiran RP. The impact of ulcerative colitis on the long-term outcome of patients with primary sclerosing cholangitis. Aliment Pharmacol Ther 2012;35:1045-1053.

35. Yanai H, Matalon S, Rosenblatt A, et al. Prognosis of primary sclerosing cholangitis in israel is independent of coexisting inflammatory bowel Disease. J Crohns Colitis 2015;9:177-184.

36. Fevery J, Van Steenbergen W, Van Pelt J, et al. Patients with largeduct primary sclerosing cholangitis and Crohn's disease have a better outcome than those with ulcerative colitis, or without IBD. Aliment Pharmacol Ther 2016;43:612-620.

37. Weismüller TJ, Trivedi PJ, Bergquist A, et al; International PSC Study Group. Patient age, sex, and inflammatory bowel disease phenotype associate with course of primary sclerosing cholangitis. Gastroenterology 2017;152:1975-1984.

38. Lindström L, Jørgensen KK, Boberg KM, et al. Risk factors and prognosis for recurrent primary sclerosing cholangitis after liver transplantation: a Nordic Multicentre Study. Scand J Gastroenterol 2018;53:297-304.

39. Hov JR, Boberg KM, Karlsen TH. Autoantibodies in primary 
sclerosing cholangitis. World J Gastroenterol 2008;14:3781-3791.

40. Roozendaal C, de Jong MA, van den Berg AP, van Wijk RT, Limburg PC, Kallenberg CG. Clinical significance of antineutrophil cytoplasmic antibodies (ANCA) in autoimmune liver diseases. J Hepatol 2000;32:734-741.

41. Terjung B, Spengler U, Sauerbruch T, Worman HJ. "Atypical p-ANCA" in IBD and hepatobiliary disorders react with a 50-kilodalton nuclear envelope protein of neutrophils and myeloid cell lines. Gastroenterology 2000;119:310-322.

42. Mitsuyama K, Niwa M, Takedatsu H, et al. Antibody markers in the diagnosis of inflammatory bowel disease. World J Gastroenterol 2016;22:1304-1310.

43. Targan SR, Landers C, Vidrich A, Czaja AJ. High-titer antineutrophil cytoplasmic antibodies in type-1 autoimmune hepatitis. Gastroenterology 1995;108:1159-1166.

44. Seibold F, Weber P, Schöning A, Mörk H, Goppel S, Scheurlen M. Neutrophil antibodies (pANCA) in chronic liver disease and inflammatory bowel disease: do they react with different antigens? Eur J Gastroenterol Hepatol 1996;8:1095-1100.

45. Hoie O, Aamodt G, Vermeire S, et al. Serological markers are associated with disease course in ulcerative colitis. A study in an unselected population-based cohort followed for 10 years. J Crohns Colitis 2008;2:114-122.

46. Ruemmele FM, Targan SR, Levy G, Dubinsky M, Braun J, Seidman EG. Diagnostic accuracy of serological assays in pediatric inflammatory bowel disease. Gastroenterology 1998;115:822-829.

47. Loly C, Belaiche J, Louis E. Predictors of severe Crohn's disease. Scand J Gastroenterol 2008;43:948-954.

48. Jostins L, Ripke S, Weersma RK, et al; International IBD Genetics Consortium (IIBDGC). Host-microbe interactions have shaped the genetic architecture of inflammatory bowel disease. Nature 2012;491:119-124.

49. Liu JZ, van Sommeren S, Huang H, et al; International IBD Genetics Consortium. Association analyses identify 38 susceptibility loci for inflammatory bowel disease and highlight shared genetic risk across populations. Nat Genet 2015;47:979-986.

50. Duboc H, Rajca S, Rainteau D, et al. Connecting dysbiosis, bileacid dysmetabolism and gut inflammation in inflammatory bowel diseases. Gut 2013;62:531-539.

51. Greve JW, Gouma DJ, Buurman WA. Bile acids inhibit endotoxininduced release of tumor necrosis factor by monocytes: an in vitro study. Hepatology 1989;10:454-458.

52. Calmus Y, Guechot J, Podevin P, Bonnefis MT, Giboudeau J, Poupon R. Differential effects of chenodeoxycholic and ursodeoxycholic acids on interleukin 1, interleukin 6 and tumor necrosis factor-alpha production by monocytes. Hepatology 1992;16:719-723.

53. Bernstein C, Holubec H, Bhattacharyya AK, et al. Carcinogenicity of deoxycholate, a secondary bile acid. Arch Toxicol 2011;85:863-871.

54. Bernstein H, Bernstein C, Payne CM, Dvorakova K, Garewal H. Bile acids as carcinogens in human gastrointestinal cancers. Mutat Res 2005;589:47-65.

55. Torres J, Palmela C, Brito H, et al. The gut microbiota, bile acids and their correlation in primary sclerosing cholangitis associated with inflammatory bowel disease. United European Gastroenterol J 2018;6:112-122.

56. Tsaitas C, Semertzidou A, Sinakos E. Update on inflammatory bowel disease in patients with primary sclerosing cholangitis. World J Hepatol 2014;6:178-187.

57. Chapman R, Cullen S. Etiopathogenesis of primary sclerosing cholangitis. World J Gastroenterol 2008;14:3350-3359.

58. Chapman R, Fevery J, Kalloo A, et al; American Association for the Study of Liver Diseases. Diagnosis and management of primary sclerosing cholangitis. Hepatology 2010;51:660-678.

59. Christensen B, Micic D, Gibson PR, et al. Vedolizumab in patients with concurrent primary sclerosing cholangitis and inflammatory bowel disease does not improve liver biochemistry but is safe and effective for the bowel disease. Aliment Pharmacol Ther 2018;47:753-762.

60. Grant AJ, Lalor PF, Hübscher SG, Briskin M, Adams DH. MAdCAM-1 expressed in chronic inflammatory liver disease supports mucosal lymphocyte adhesion to hepatic endothelium (MAdCAM-1 in chronic inflammatory liver disease). Hepatology 2001;33:1065-1072.

61. Vermeire S, Sandborn WJ, Danese S, et al. Anti-MAdCAM antibody (PF-00547659) for ulcerative colitis (TURANDOT): a phase 2, randomised, double-blind, placebo-controlled trial. Lancet 2017;390:135-144.

62. Cesaro C, Tiso A, Del Prete A, et al. Gut microbiota and probiotics in chronic liver diseases. Dig Liver Dis 2011;43:431-438.

63. Tabibian JH, Varghese C, LaRusso NF, O'Hara SP. The enteric microbiome in hepatobiliary health and disease. Liver Int 2016;36:480-487.

64. Yamada S, Ishii M, Liang LS, Yamamoto T, Toyota T. Small duct cholangitis induced by $\mathrm{N}$-formyl L-methionine L-leucine L-tyrosine in rats. J Gastroenterol 1994;29:631-636.

65. Färkkilä M, Karvonen AL, Nurmi $\mathrm{H}$, et al. Metronidazole and ursodeoxycholic acid for primary sclerosing cholangitis: a randomized placebo-controlled trial. Hepatology 2004;40:1379-1386.

66. Quraishi MN, Sergeant M, Kay G, et al. The gut-adherent microbiota of PSC-IBD is distinct to that of IBD. Gut 2017;66:386-388.

67. Kummen M, Holm K, Anmarkrud JA, et al. The gut microbial profile in patients with primary sclerosing cholangitis is distinct from patients with ulcerative colitis without biliary disease and healthy controls. Gut 2017;66:611-619.

68. Palmela C, Peerani F, Castaneda D, Torres J, Itzkowitz SH. Inflammatory bowel disease and primary sclerosing cholangitis: a review of the phenotype and associated specific features. Gut Liver 2018;12:17-29.

69. Lv LX, Fang DQ, Shi D, et al. Alterations and correlations of the gut microbiome, metabolism and immunity in patients with primary biliary cirrhosis. Environ Microbiol 2016;18:2272-2286.

70. Chen Y, Ji F, Guo J, Shi D, Fang D, Li L. Dysbiosis of small intestinal microbiota in liver cirrhosis and its association with etiology. Sci Rep 2016;6:34055.

71. Wei X, Yan X, Zou D, et al. Abnormal fecal microbiota community and functions in patients with hepatitis B liver cirrhosis as revealed by a metagenomic approach. BMC Gastroenterol 2013;13:175.

72. Söderlund S, Brandt L, Lapidus A, et al. Decreasing time-trends of colorectal cancer in a large cohort of patients with inflammatory bowel disease. Gastroenterology 2009;136:1561-1567.

73. Rutter M, Saunders B, Wilkinson K, et al. Severity of inflammation is a risk factor for colorectal neoplasia in ulcerative colitis. Gastroenterology 2004;126:451-459.

74. Annese V, Daperno M, Rutter MD, et al; European Crohn's and Colitis Organisation. European evidence based consensus for endoscopy in inflammatory bowel disease. J Crohns Colitis 2013;7:982-1018.

75. Jess T, Loftus EV Jr, Velayos FS, et al. Risk factors for colorectal neoplasia in inflammatory bowel disease: a nested case-control study from Copenhagen county, Denmark and Olmsted county, Minnesota. Am J Gastroenterol 2007;102:829-836.

76. Eaden JA, Abrams KR, Mayberry JF. The risk of colorectal cancer in ulcerative colitis: a meta-analysis. Gut 2001;48:526-535.

77. Canavan C, Abrams KR, Mayberry J. Meta-analysis: colorectal and small bowel cancer risk in patients with Crohn's disease. Aliment Pharmacol Ther 2006;23:1097-1104.

78. Harbord M, Annese V, Vavricka SR, et al; European Crohn's and Colitis Organisation. The first European evidence-based consensus on extra-intestinal manifestations in inflammatory bowel disease. 
J Crohns Colitis 2016;10:239-254.

79. Aabakken L, Karlsen TH, Albert J, et al. Role of endoscopy in primary sclerosing cholangitis: European Society of Gastrointestinal Endoscopy (ESGE) and European Association for the Study of the Liver (EASL) Clinical Guideline. Endoscopy 2017;49:588-608.

80. Lindor KD, Kowdley KV, Harrison ME; American College of Gastroenterology. ACG Clinical Guideline: primary sclerosing cholangitis. Am J Gastroenterol 2015;110:646-659.

81. Gulamhusein AF, Eaton JE, Tabibian JH, Atkinson EJ, Juran BD, Lazaridis KN. Duration of inflammatory bowel disease is associated with increased risk of cholangiocarcinoma in patients with primary sclerosing cholangitis and IBD. Am J Gastroenterol 2016;111:705-711.

82. European Association for the Study of the Liver. EASL Clinical
Practice Guidelines: management of cholestatic liver diseases. J Hepatol 2009;51:237-267.

83. Eaton JE, Thackeray EW, Lindor KD. Likelihood of malignancy in gallbladder polyps and outcomes following cholecystectomy in primary sclerosing cholangitis. Am $J$ Gastroenterol 2012;107:431-439.

84. Zenouzi R, Weismüller TJ, Hübener P, et al. Low risk of hepatocellular carcinoma in patients with primary sclerosing cholangitis with cirrhosis. Clin Gastroenterol Hepatol 2014;12:1733-1738.

85. Itzkowitz SH, Harpaz N. Diagnosis and management of dysplasia in patients with inflammatory bowel diseases. Gastroenterology 2004;126:1634-1648.

86. Ullman T, Odze R, Farraye FA. Diagnosis and management of dysplasia in patients with ulcerative colitis and Crohn's disease of the colon. Inflamm Bowel Dis 2009;15:630-638. 